\title{
El rendimiento académico: La influencia de las expectativas, el optimismo y la autoeficacia
}

\section{Academic performance: The influence of expectations, optimism and self-effectiveness}

\author{
Laura Redondo, Tania Corrás, Mercedes Novo, Francisca Fariña \\ Universidad de Vigo
}

\begin{abstract}
Resumen
El Espacio Europeo de Educación Superior exige abordar el proceso de enseñanza-aprendizaje desde el desarrollo de competencias. Para ello es necesario incluir los distintos factores psicosociales presentes en la comunidad universitaria, y su incidencia en el rendimiento académico. El objetivo del presente estudio es analizar si las expectativas, el optimismo y la autoeficacia se relacionan con el rendimiento académico. Para ello se contó con 127 estudiantes de Grado, siendo el $72.5 \%$ hombres y el $27.5 \%$ mujeres, y su media de edad de 20.14 años (DT=2.40). Se ha observado que las expectativas se relacionan con el resultado obtenido y la autoeficacia con las expectativas profesionales.

Palabras clave: Rendimiento, expectativas, optimismo,
\end{abstract} autoeficacia.

\begin{abstract}
The European Higher Education Area requires addressing the teaching-learning process from the development of competencies. To obtain this it is necessary to include the different psychosocial factors present in the higher education community, and their impact on academic performance. The objective of the present study is to analyze if expectations, optimism and self-efficacy are related to academic performance. To achieve this, there were 127 undergraduate students, $72.5 \%$ men and $27.5 \%$ women, and their mean age was 20.14 years $(S D=2.40)$. It has been observed that the expectations are related to the result obtained and the self-efficacy with the professional expectations.

Keyword: Performance, expectations, optimism, self-efficacy.
\end{abstract}

\section{Introducción}

Desde sus inicios las universidades han tenido como misión la implementación de una formación integral para sus estudiantes a través de tres grandes vertientes: el desarrollo personal, el profesional y el de ciudadanía (Villa y Pobete, 2007). Precisamente, la línea de actuación del Espacio Europeo de Educación Superior (EEES) establece cuatro ejes conductores del desarrollo de competencias a adquirir por el alumnado, poniendo en valor la adquisición de las habilidades sociales necesarias para su integración en la sociedad y su desarrollo personal: aprender a conocer, aprender a hacer, aprender a vivir juntos y aprender a ser (Delors, 2006). En este sentido, cobra especial relevancia en la planificación docente tener en cuenta las competencias psicosocioemocionales por su incidencia tanto en el rendimiento académico, como en la futura práctica profesional (Redondo, Alonso, Fariña, Cuevas y Acuña, 2017). Asimismo, la participación y actividad del estudiante en los procesos de aprendizaje y docencia pueden ser espacios adecuados para generar en ellos un sentido de pertenencia, implicación y participación genuina en la vida universitaria, potenciando así su sentido de comunidad y de compromiso (Bara y Martínez, 2012; Novo, Redondo, Seijo y Arce, 2016). Así, se hace necesario un abordaje directo de este tipo de competencias, a la vez que una evaluación efectiva de las mismas, para lo cual el rendimiento académico es uno de los indicadores de referencia dentro de nuestro sistema educativo.

Concretamente, el rendimiento es un constructo de gran complejidad que ha sufrido una importante evolución, teniendo como punto de partida los años sesenta, cuando las causas del bajo rendimiento se focalizaban casi exclusivamente en el alumnado (Bullón et al., 2017). Sin embargo, lo que indica la literatura (p.e., Proctor, Hurst, Prevant, Petscher y Adams, 2006; Sirin, 2005; Tejedor, 2003) es la existencia de numerosos factores mediadores, tanto de corte personal, entre ellos la capacidad cognitiva y la propia personalidad, como de tipo educacional; siendo ambos relevantes en el desarrollo de la identidad y la autonomía de los estudiantes (Bullón et al 2017).

Por otro lado, en el contexto universitario se ha señalado que las actividades propias de la vida académica suponen una fuente de estrés y ansiedad que pueden influir tanto en el rendimiento como en el bienestar físico y psicológico del alumnado (Martín, 2007; Misray McKean, 2000). En consecuencia, cobran especial relevancia aquellas variables que sirven de factores protectores o neutralizadores del estrés o situaciones estresantes. En este sentido, destacan las habilidades en resolución de problemas, determinados factores de personalidad, un buen desarrollo de habilidades sociales (Bandura, 1992; Caballo, 1997; Londoño, 2007; Taylor, 
2000), la eficacia personal (Lazarus y Folkman, 1986; Martin, 2002), el afrontamiento positivo o centrado en el problema (Gordon, Feldman, Crose y Schoenal, 2002; Martínez-Correa, Reyes del Paso, García-León y González-Jareño, 2006), el soporte social real y percibido (Pratt et al., 2000), o el optimismo. Este último se considera como un propulsor de conductas de afrontamiento adaptativas (Scheier y Carver, 1985), tratándose de una tendencia disposicional a mantener expectativas positivas generalizadas, incluso cuando los individuos se enfrentan a la adversidad o a dificultades en sus vidas (González y Extremera, 2010; Scheier, Carver y Bridges, 2001). De esta manera, se puede entender como un rasgo de personalidad de la dimensión optimismo-pesimismo (Ferrando, Chico y Tous, 2002). Particularmente, con universitarios se encontró que el optimismo es determinante para la conducta de los mismos con respecto a su permanencia en la titulación, el no renunciar a convocatorias y la superación de las materias (Londoño, 2009). Otra de las variables que se ha relacionado con el rendimiento es el de las expectativas (Garreta, 2007), ya sean las generadas por el propio individuo como por sus personas de referencia. En esta línea, Merton (1957) ya establecía la existencia de un fenómeno que acuñó como profecía autocumplida(PA), donde la expectativa de que un evento o suceso ocurra aumenta las posibilidades de que efectivamente suceda. Por su parte, Rosenthal y Jacobson (1968) pusieron de relevancia lo que denominaron efecto Pigmalión, en el que las expectativas y creencias de un tercero acerca de algo o alguien, sean positivas o negativas, conducen inconsciente o involuntariamente al resultado. Más recientemente, Rovira (2006) ha subrayado la influencia de las percepciones trasmitidas de los progenitores a sus hijas y/o hijos, y las del profesorado al alumnado. Por ende, no es de extrañar que las expectativas se consideren una de las variables de carácter psicoemocional más importantes en relación al rendimiento, especialmente las del profesorado respecto al alumnado existiendo evidencia de su correlación directa con el desempeño de los estudiantes (Duseky Joseph, 1983; Tsiplakides y Keramida, 2010).

Teniendo en cuenta lo anterior, este trabajo forma parte de un estudio global sobre cómo distintas variables psicoemocionales se relacionan o median el rendimiento académico del alumnado en la enseñanza superior. Concretamente, la investigación que se plantea tiene como objetivo estudiar si las expectativas, el optimismo y la autoeficacia se relacionan con el rendimiento académico, así como la concordancia entre la predicción del rendimiento y el finalmente obtenido.

\section{Método}

\section{Participantes}

La muestra está compuesta por 127 estudiantes universitarios, que estaban en el segundo semestre del primer curso de la titulación de Ciencias de la Actividad Física y del Deporte. El 72.5\% eran hombres y el $27.5 \%$ mujeres, siendo su media de edad era de 20.14 años (DT=2.40).

\section{Procedimiento}

El primer día de clase de la materia de psicología se informó al alumnado que se estaba realizando un estudio sobre las expectativas y el rendimiento. Se les explicó que quienes participaban tenían que cumplimentar un cuestionario en el que se evaluaban diferentes variables psicoemocionales, y además tenían que informar sobre sus expectativas sobre la asignatura, en cuanto a la nota que iba a obtener, el grado de aprendizaje que iban adquirir, y la utilidad de éste para su futura práctica profesional. Tras lo cual, se les solicitó su participación voluntaria en el mismo, asegurándoles que todos los datos se anonimizarán y no tendrían repercusión en su nota final de la materia, puesto que estos se analizarían tras entregar las calificaciones.

\section{Instrumentos de medida}

Se aplicó un cuestionario ad hoc, en el que se preguntaba por la nota que pensaban obtener a final de curso en esta signatura, en un intervalo de 1 a 10. Además se pedía que se señalaran, las expectativas con respecto al nivel de aprendizaje y la utilidad en la futura práctica profesional, en una escala Likert con cinco opciones, donde 1= muy baja, 2 = baja, 3= media, 4=alta, y $5=$ muy alta.

Para medir el optimismo se utilizó la versión española del LOT-R (Otero, Luengo, Romero, Gómez y Castro, 1998), en cuya versión original se obtuvo una consistencia interna de .78 (Scheier et al., 1994). En su adaptación española los resultados psicométricos hallados indican esta versión de LOT-R mantiene propiedades muy similares a las de la versión original (Ferrando, Chico, y Tous, 2002). La escala cuenta con un formato de respuesta tipo Likert con siete opciones, donde $1=$ no estoy de acuerdo, $2=$ estoy un poco desacuerdo, $3=$ estoy un poco de acuerdo, 4= estoy de acuerdo, 5= estoy bastante de acuerdo, 6= estoy тиу de acuerdo y $7=$ estoy totalmente de acuerdo.

Se administró la escala de autoeficacia general de Baessler y Schwarcer (1996) para medir el nivel de eficacia percibida por el alumnado. La escala cuenta con una consistencia interna de .87 (Suárez, García y Moreno, 2000). El formato de respuesta de la escala es de tipo Likert de 1 a 10 desde 1=totalmente en desacuerdo, hasta $10=$ totalmente de acuerdo.

Además, se registró las notas del examen, de la parte práctica, el trabajo en grupo, así como la nota global de la asignatura.

\footnotetext{
Análisis de datos

Se ha realizado un análisis a nivel descriptivo, y un análisis correlacional bivariado entre las distintas valoraciones obtenidas y las expectativas sobre la nota, sobre el aprendizaje adquirido y sobre la utilidad en la práctica profesional futura. Por otro lado, se ha realizado un análisis estadístico con la prueba $t$ de student para muestras relacionadas, para analizar si existen diferencias significativas entre las expectativas de nota y la calificación obtenida de manera global, así como en las distintas áreas de evaluación (examen, trabajo en grupo y prácticas).
} 


\section{Resultados}

El análisis de los resultados a nivel descriptivo (Ver Tabla 1) muestra una tendencia a pronosticar una calificación global más elevada a la que se alcanza finalmente. En el análisis pormenorizado del rendimiento, se observa una predisposición a aspirar a una nota más alta que la que finalmente se obtiene en el examen teórico, si bien los resultados en el trabajo en grupo y en la parte práctica de la materia son más elevados que las expectativas que tenía el alumno al inicio del curso.

Tabla 1.

Estadísticos descriptivos del rendimiento académico, expectativas, nivel de optimismo y nivel de autoeficacia.

\begin{tabular}{lcc}
\hline & $M$ & $D T$ \\
\hline Calificación Total Obtenida & 6.32 & 1.97 \\
Calificación del Examen & 4.86 & 2.25 \\
Calificación del Trabajo en Grupo & 7.45 & 2.74 \\
Calificación de Prácticas & 8.80 & 0.62 \\
Expectativa de Nota & 7.46 & 1.13 \\
Expectativa de Aprendizaje & 3.87 & 0.81 \\
Expectativa de Utilidad Práctica & 4.01 & 0.85 \\
Escala LOT-R & 33.26 & 4.48 \\
Escala de Autoeficacia General & 99.54 & 36.48 \\
\hline
\end{tabular}

Los resultados del análisis correlacional sobre el rendimiento académico muestran que la expectativa de nota correlaciona positivamente con la calificación final obtenida $(r=.38 ; p<.01)$, así como con la calificación del examen $(r=.33 ; p<.01)$ y con la del trabajo en grupo $(r=.30 ; p<.01)$. Si bien no se encuentra relación de esta con la calificación obtenida en la parte práctica de la materia. Cabe señalar que la expectativa de utilidad práctica se correlaciona con la calificación del trabajo en grupo $(r=.29 ; p<.01)$. En relación a la interacción entre las expectativas, se ha hallado una relación directa y positiva de la expectativa de nota con la expectativa de aprendizaje $(r=.27 ; p<.05)$, y a la expectativa de utilidad práctica $(r=.31 ; p<.01)$. Respecto al sentimiento de autoeficacia, éste correlaciona con la expectativa de utilidad práctica $(r=.29, p<.05)$ y con la expectativa de aprendizaje $(r=.43 ; p<.01)$. En cuanto a la variable optimismo este guarda una relación positiva con la expectativa de utilidad práctica de la materia $(r=.28$; $p<.05)$, no correlacionando con ninguna otra variable de las registradas en el cuestionario.

Por otro lado, el análisis de datos muestra que la calificación de la expectativa de nota $(M=7.44)$ es mayor que la obtenida $(M=6.46)$, y que la alcanzada en el examen teórico $(M=4.78), t(126)=10.98, p<.001$, en tanto que es menor que la obtenida en el trabajo en grupo $(M=8.82), t(126)=-9.06, p<.001, \mathrm{y}$ en las prácticas $(M=$ $8.04), t(126)=2.58, p<.05$.

\section{Conclusiones y Discusión}

A la luz de los datos obtenidos en relación al rendimiento académico, se puede concluir que las expectativas se relacionan con el resultado, alcanzando una mayor calificación aquellos que se fijaron unas expectativas más elevadas, en la línea de lo que ya planteaban Dusek y Joseph (1983) o Rosenthal y
Jacobson (1968). De manera particular, se aprecia que las expectativas son más elevadas en relación al examen respecto a las de la parte práctica y de trabajo grupal de la materia. Cabe destacar que el papel de las expectativas sobre la utilidad práctica tiene un papel determinante en el rendimiento de los trabajos en grupo. Respecto a la interacción entre expectativas, se puede concluir que la expectativa de aprendizaje y la expectativa de utilidad práctica aumentan la expectativa de la nota final a obtener.

Dada la importancia de las expectativas sobre el rendimiento académico, en línea con lo planteado por Weiner $(1979,1985,1986)$ se mostró que los estudiantes realizan para las materias distintas interpretaciones y valoraciones sobre los resultados que obtendrán, y esto afecta a su rendimiento. Resta conocer cuál es la atribución que el alumnado hace de este resultado y su impacto en su bienestar físico/psicológico (Aranceli, Perea y Ormeño, 2006; Guarino, Gavidia, Antor y Caballero, 2000), y en su rendimiento (De Pablo, Subirá, Martín, de Flores y Valdés, 1990; Martín, 2007; Misra y McKean, 2000; Struthers, Perry y Menec, 2000). Los Planes de Acción Tutorial podrían utilizarse para averiguarlo, y de ser necesario intervenir para minimizar el riesgo de desajuste cognitivo o académico.

En relación al sentimiento de autoeficacia, se encontró una relación con las expectativas profesionales, en línea con otras investigaciones (Felneret al., 1995; Harter, 1993; Hernández-Guzmán y Sánchez-Sosa, 1996) que establecen su relación con el rendimiento académico del alumnado.

Por otra parte, respecto al optimismo los resultados indican que está relacionado con la expectativa de utilidad práctica de la materia; sin embargo, en otro estudio (Redondo, Alonso, Fariña, Cuevas y Acuña, 2017) hemos encontrado que se relacionaba con la expectativa de nota. Esto podría ser debido a variables moderadoras como la titulación o la propia materia puesto que aquello que se percibe como un mayor reto será hacia lo que el optimismo se movilice como recurso de afrontamiento (González y Extremera, 2010; Scheier, Carver y Bridges, 2001). En este sentido, se hace necesario ahondar en esta cuestión en futuros estudios.

En suma, como indica Delors (2006) se pone de relevancia la necesidad de conocer las variables que median en el proceso de enseñanza-aprendizaje para poder optimizar el rendimiento y el desarrollo integral del alumnado.

\section{Agradecimientos}

Este trabajo se enmarca dentro del Contrato Programa 2017 de la Universidad de Vigo.

\section{Referencias}

Aranceli, S., Perea, P. y Ormeño, R. (2006). Evaluación de niveles, situaciones generadoras y manifestaciones de estrés académico en alumnos de tercer y cuarto año de una Facultad de Estomatología. Revista Estomatológica Herediana, 16, 15-20.

Bandura, A. (1992). A self-efficacy mechanism in psycholbiologic functioning. En R. Schwarzer (Ed.), 
Self-efficacy: thougt control of action (pp. 357-394). Washington USA: Hemisphere.

Bara, F. E., y Martínez, M. (2012). ¿Son universidades todas las universidades? La universidad como comunidad ética. Bordón, 64(3), 77-92.

Baessler, J. y Schwarcer, R. (1996). Evaluación de la autoeficacia: Adaptación española de la escala de Autoeficacia General. Ansiedad y Estrés, 2, 1-8.

Bullón, F. F., Campos, M. M., Castaño, E. F., del Barco, B. L., y del Río, M. I. P. (2017). Análisis del rendimiento académico de los alumnos de educación secundaria obligatoria según las variables familiares. Educación XX1, 20.

Caballo, V. (1997). Manual para el tratamiento cognitivo conductual de los trastornos psicológicos. Madrid España: SigloXXI.

De Pablo, J., Subirá, S., Martín, M., de Flores, T. y Valdés, M. (1990). Examination-associated Anxiety in Students of Medicine. Academic Medicine, 65(11), 706-707.

Delors, J. (2006). La educación encierra un tesoro: Informe a la UNESCO de la Comisión Internacional sobre la Educación para el siglo XXI.Madrid: Santillana.

Dusek, J. B., y Joseph, G. (1983). The bases of teacher expectancies: A meta-analysis. Journal of Educational Psychology, 75(3), 327.

Feldman, L., Goncalves, L., Chacón-Puignau, G., Zaragoza, J., Bagés, N., y De Pablo, J. (2008). Relaciones entre estrés académico, apoyo social, salud mental y rendimiento académico en estudiantes universitarios Psychologica, 7(3), 739-751.

Ferrando, P., Chico, E. y Tous, J. (2002). Propiedades psicométricas del Test de Optimismo. Life Orientation Test. Psichotema, 14, 673-680.

Garreta, J. (Ed). (2007). La relación familia-escuela. Lleida: Editions de la Universidad de Lleida.

González, V., y Extremera, N. (2010). Daily life activities as mediators of the relationship between personality variables and subjective well-being among older adults. Personality and Individual Differences, 49, 124-129.

doi: 10.1016/j.paid.2010.03.019

Gordon, A., Feldman, D.; Crose, R. y Schoen, E. (2002).The paper of the religious belief in facing chronic disease. Counseling and Values, 46(3) 162174.

Guarino, L., Gavidia, I., Antor, M. y Caballero, H. (2000). Estrés, salud mental y cambios inmunológicos en estudiantes universitarios. Psicología Conductual, 8, 57-71.

Harter, S. (1993). Causes and consequences of low self-esteem in children and adolescents. En R. F. Baumeister (Ed.), Self-esteem: The puzzle of low self-regard(pp. 87-116). New York: Plenum Press.

Hernández-Guzmán, L. y Sánchez-Sosa, J. J. (1996). Factores de riesgo y protectores que afectan el aprovechamiento escolar. Psicología y problemática social. México: Universidad Nacional Autónoma de México.
Lazarus, R. y Folkman, S. (1986). Estrés y procesos cognitivos. Barcelona España: Martínez Roca.

Londoño, C. (2009). Optimismo y salud positiva como predictores de la adaptación a la vida universitaria. Universidad Católica de Colombia. Acta colombiana de Psicología, 12, 95-107.

Londoño, C. (2007). Construcción de un modelo cognitivo social integrado para la prevención del abuso de alcohol en universitarios bogotanos. Tesis de maestría, Facultad de Psicología, Universidad Nacional de Colombia.

Martin, N. (2002). Feeling optimistic? Psychologist, 15(6), 309-321.

Martín, I. (2007). Estrés académico en estudiantes universitarios. Apuntes de Psicología, 25, 87-99.

Merton, R. K. (1957). Social Theory and Social Structure. New York: Free Press.

Misra, R. y McKean, M. (2000). College Student's Academic Stress and Its Relation to Their Anxiety, Time Management and Leisure Satisfaction. American Journal of HealthStudies, 16, 41-52.

Novo, M., Redondo, L., Seijo, D., y Arce, R. (2016). Diseño y validación de una escala para la evaluación del sentido de comunidad en grupos académicos virtuales. Revista de Investigación en Educación, 14(2), 126-140.

Otero, J. M., Luengo, A., Romero, F., Gómez, J. A. y Castro, C. (1998). Psicología de la Personalidad. Manual de Prácticas. Barcelona: Ariel Practicum.

Pratt, M., Bowers, C., Terzjan, B. y Hunsberger, B. (2000). To facilitate the transition to the university: evaluation of a social program of intervention of and discussion. Journal of College Student Development, 41(4), $427-435$.

Proctor, B., Hurst, A., Prevant, F., Petscher, Y., y Adams, K. (2006). Study skills profiles of normal-achieving and academically struggling college students. Journal of College Student Development, 47, 37-51.

Redondo, L., Alonso, A., Fariña, F., Cuevas, M., y Acuña, A. (junio, 2017). Expectativas de rendemento e aprendizaxe: $\mathrm{O}$ peso do optimismo. $\mathrm{V}$ Conferencia Internacional e Nacional de Docencia Universitaria (CINDU). Vigo, Pontevedra.

Rosenthal, R. y Jacobson, L. (1968). Pygmalion en la escuela. Expectativas del maestro y desarrollo intelectual del alumno. Madrid: Marova.

Rovira, A. (2006). Superarse con el efecto Pigmalión. Revista de educación, 340, 379-414.

Scheier, M., y Carver, C. (1985). Optimism, coping and health: Assessment and implications of generalized outcome expectancies. Health Psychology, 4, 219-247.

Schier, M. F., Carver, C. S., y Bridges, M. W. (1994). Distinguishing optimism from neuroticism (and trait anxiety, Self-mastery, and Self-esteem): a reevaluating of the life oriental test. Journal of Personality and Social Psychology, 67(6), 1063-1078.

Scheier, M. F., Carver, C. S., y Bridges, M. W. (2001). Optimism, pessimism, and psychological well-being. En E. C. Chang (Ed.), Optimism and pessimism: Implications for theory, research, and practice (pp. 189-216). Washington, DC, EEUU: American Psychological Association. 
Sirin, S. R. (2005). Socioeconomic status and academic achievement: a meta-analytic review of research. Review of Educational Research, 75, 417-453.

Struthers, C., Perry, R. y Menec, V. (2000). An Examination of the Relationship among Academic Stress, Coping, Motivation and Performance at College. Research in Higher Education, 41, 579-590.

Suárez, P. S., García, A. M. P., y Moreno, J. B. (2000). Escala de autoeficacia general: datos psicométricos de la adaptación para población española. Psicothema, 12(Suplemento), 509-513.

Taylor, M. J. (2000). The influence of self-efficacy on alcohol use among American indians. Cultural Diversity and Ethnic Minority Psychology, 6(2), 152-167.

Tejedor, F. J. (2003). Poder explicativo de algunos determinantes del rendimiento en los estudios universitarios. Revista Española de Pedagogía, 61, 5-32.

Villa, A., y Poblete, M. (2007). Aprendizaje basado en competencias. Una propuesta para la evaluación de las competencias genéricas. Bilbao: Mensajero/ICE Universidad de Deusto.

Weiner, B. (1979): A theory of motivation for some classroom experiences. Journal of Educational Psychology, 71, 3-25.

Weiner, B. (1985). An attributional theory of achievement motivation and emotion. Psychological Review, 92, 548-573.

Weiner, B. (1986). An atributional theory of motivation and emotion. New York: Springer-Verlag. 